\title{
LncRNA MALAT1 promotes osteoarthritis by modulating miR-150-5p/AKT3 axis
}

\author{
Ying Zhang*, Fuyou Wang, Guangxing Chen, Rui He and Liu Yang
}

\begin{abstract}
Background: Many studies have reported that long noncoding RNAs (IncRNAs) could act as sponges for microRNAs (miRNAs) and play important roles in the regulation of osteoarthritis (OA). Yet, the underlying mechanisms of IncRNA metastasis-associated lung adenocarcinoma transcript 1 (MALAT1) in OA are still unclear. Therefore, we aimed to explore the regulation mechanisms of MALAT1 in OA procession.

Methods: IL-1 $\beta$ treatment in chondrocyte was used to mimic OA in vitro. MALAT1, miR-150-5p and AKT3 expression levels were detected via qRT-PCR. The protein levels of AKT3, MMP-13, ADAMTS-5, Bax, BCl-2, cleaved-PARP, collagen II and aggracan were measured by western blot. MTT assay was performed to detect cell proliferation ability. The apoptosis of chondrocytes was determined using flow cytometry and western blot. Luciferase assay and RNA immunoprecipitation (RIP) assays were used to confirm the relationship among MALAT1, miR-150-5p and AKT3.

Results: In our study, MALAT1 and AKT3 were upregulated while miR-150-5p was downregulated in OA in vitro and vivo. The level of miR-150-5p was negatively correlated with that of MALAT1 or AKT3. More importantly, overexpression of MALAT1 promoted the expression of AKT3 by negatively regulating miR-150-5p. MALAT1 knockdown inhibited cell proliferation, promoted apoptosis, increased MMP-13, ADAMTS-5 expression and decreased collagen II, aggracan expression in IL-1 $\beta$ treated chondrocytes. MALAT1 upregulation or AKT3 overexpression enhanced proliferation, inhibited apoptosis and extracellular matrix (ECM) degradation, which was undermined by overexpression of miR-150-5p. By contrast, miR-150-5p depletion rescued the effect of MALAT1 downregulation or loss of AKT3 on IL-1 $\beta$-stimulated chondrocytes.
\end{abstract}

Conclusion: MALAT1 was responsible for cell proliferation, apoptosis, and ECM degradation via miR-150-5p/AKT3 axis.

Keywords: OA, MALAT1, miR-150-5p, AKT3, MALAT1/miR-150-5p/AKT3

\section{Background}

Osteoarthritis $(\mathrm{OA})$ is the most common type of joint disease and is considered be a disease involving all tissues of the joint [1-3]. The main feature of OA is the destruction of articular cartilage caused by the imbalance of extracellular matrix (ECM) components [4]. There are two main reasons for the imbalance of ECM. One is the degradation of ECM, and the other is the formation of cartilage tissue [5]. Most people over 65 and about $80 \%$ of people over 75 have OA [2]. OA can cause severe physical

*Correspondence: wmlch752@sina.com

Center for Joint Surgery, Southwest Hospital, The Third Military Medical University (Army Medical University), 30 Gaotanyan Main St., Shapingba Dist., Chongqing 400038, People's Republic of China disability, pain, stiffness and loss of mobility, leading to obstacles in the daily activities of patients [4]. Although there are many treatments including drug treatment, acupuncture therapy, electromagnetic therapy, stem cell treatment [3,6-13]. OA is still difficult to cure. Therefore, it is necessary to explore the deep molecular mechanisms of OA.

Many studies have shown that long noncoding RNAs (lncRNAs) are involved in cell biology processes such as cell proliferation and apoptosis, cell differentiation, tumorigenesis and metastasis [14-16]. Alterations in lncRNAs can lead to a variety of abnormal expressions of genes associated with disease and biological functions [17]. In a previous study, lncRNA FOXD2-AS1 modulated Cyclin 
D1 expression by sponging miR-206, resulting in regulating chondrocytes proliferation in OA [18]. LncRNA PVT1 could sponge for miR-488-3p and regulate chondrocyte apoptosis in OA [19]. LncRNA small nucleolar RNA host gene 5 (SNHG5) promotes the proliferation of chondrocytes via miR-26a/SOX2 signal axis in OA [20]. LncRNA-CIR enhances articular cartilage degeneration in OA through regulating autophagy [21]. However, the underlying mechanisms of action of lncRNA MALAT1 in OA are still lacking. MicroRNAs (miRNAs), a series of small noncoding RNAs with 18-22 nts in length, have been reported to be interacted with lncRNAs to exert its biological function [22, 23]. For example, miR-630 promotes epithelial ovarian cancer proliferation and invasion via targeting Krüppel-like factor 6 [24]. Many studies also have reported the roles of miR-150-5p. miR-150-5p is also involved in the linc00673 depletion-mediated the proliferation, migration, invasion and EMT suppressing effect in non-small cell lung cancer [25]. A previous study also indicated that miR-150-5p enhanced cell apoptosis in paclitaxel-resistant ovarian cancer cells by targeting Notch3 [26]. Although many researchers have investigated the function of miR-150-5p in many cancers [27, 28], its role in OA remains largely unknown.

In the present study, we sought to explore the function of MALAT1 and its underlying mechanisms in OA. In addition, whether miR-150-5p and AKT3 is associated with the function of MALAT1 is also addressed.

\section{Materials and methods}

\section{Clinical samples of cartilage tissues}

OA cartilage tissues were obtained from OA patients underwent total knee replacement surgery $(n=42,22$ men and 20 women, age range $59-70$ years). Normal cartilage tissues were acquired from patients underwent the amputation without OA or rheumatoid arthritis history $(\mathrm{n}=20,10$ men and 10 women, age range $38-47$ years). All cartilage tissues conformed to the diagnostic criteria of osteoarthritis of the Orthopaedic Society of the Chinese Medical Association. Cartilage tissue samples were immediately frozen in the liquid nitrogen for storage after surgery. This study was approved by the Research Ethics Committee of Southwest Hospital, the Third Military Medical University (Army Medical University). And all the donors and their families read and signed the informed consents.

\section{Cell culture}

The excess fibrous connective tissue in the normal cartilage tissues was subtracted, and the cartilage tissue was cut into about $1 \mathrm{~mm}^{3}$ size. After washing with PBS containing penicillin sodium and gentamicin, fivefold volume of $0.25 \%$ trypsin was added, digested in incubator at
$37{ }^{\circ} \mathrm{C}$ for $30 \mathrm{~min}$, discarded supernatant; fivefold volume of $0.2 \%$ Collagenase type II was added, digested in $37^{\circ} \mathrm{C}$ incubator for $16 \mathrm{~h}$, and cells were collected every $4 \mathrm{~h}$. The cell suspension was filtered and digested by 200 mesh filter at $1000 \mathrm{r} / \mathrm{min}$. After centrifugation for $5 \mathrm{~min}$, the supernatant was discarded. The precipitation was washed 3 times with complete culture medium containing 10\% fetal bovine serum. Finally, the cells were inoculated in a culture flask at a density of $1 \times 10^{5} / \mathrm{mL}$ and cultured in the incubator containing $5 \% \mathrm{CO}_{2}$ at $37^{\circ} \mathrm{C}$. Small interfering RNA for MALAT1 (si-MALAT1) and si-MALAT1 scramble (si-NC), MALAT1 overexpression plasmid or pcDNA 3.0 (NC), miR-150-5p mimic (miR-150-5p) or negative control mimic (miR-NC), miR-150-5p inhibitor (anti-miR-150-5p) or negative control inhibitor (antimiR-NC), and si-AKT3 or AKT3 overexpression plasmid were transfected into chondrocytes. After $48 \mathrm{~h}$ of transfection, chondrocytes were stimulated with IL-1 $(10 \mathrm{ng} /$ $\mathrm{mL}$; Sigma, St. Louis, MO, USA) for the $24 \mathrm{~h}$ and used for further analysis.

All the experiments were done with the second and third subculture of chondrocytes. All operations are performed under sterile conditions.

\section{qRT-PCR}

Total RNAs were isolated from human cartilage tissues and cultured chondrocytes using the TRIzol reagent. Isolated RNAs were reverse-transcribed with the use of a kit (Promega). Quantitative real-time PCR (qRTPCR) was performed on the Biosystems 7300 Real-Time PCR system (ABI, Foster City, CA, USA) by using SYBR GreenMix (Takara). $\Delta \Delta \mathrm{Ct}$ method was used to calculate the relative gene expression. U6 expression was used as the internal control for IncRNA and miRNA expression. $\beta$-Actin was used to normalize the mRNA expression level.

\section{Western blot}

Total proteins were extracted by lysing cells on ice with RIPA lysis buffer (Pierce, Rockford, IL, US) for $15 \mathrm{~min}$. The protein concentration was measured by the BCA Assay Kit (Thermo Scientific). Protein fractions were separated with sodium dodecyl sulfate-polyacrylamide gel electrophoresis gels $(10 \%)$ and then transferred onto polyvinylidene difluoride (PVDF) membrane (Millipore, Bedford, MA, USA). After blocked with 5\% BSA for $2 \mathrm{~h}$ at $4{ }^{\circ} \mathrm{C}$, membranes were incubated with specific primary antibodies anti-AKT3, anti- $\beta$-actin, anti-MMP-13, antiADAMTS-5, anti-collagen II and anti-aggrecan (1:1000 dilution; Abcam) overnight at $4{ }^{\circ} \mathrm{C}$. The membranes were washed thrice and then incubated with horseradish peroxidase (HRP)-conjugated secondary anti-rabbit antibodies for $1 \mathrm{~h}$. All protein bands were detected by ECL 
detection kit (Thermo, Waltham, MA, USA) and ChemiDoc XRS System (Bio-Rad, Hercules, CA, USA). $\beta$-Actin was used as an internal normalization control.

\section{MTT assay}

The growing conditions of cells were examined by MTT assay. The cells grown in logarithmic phase were inoculated into a 96-well culture plate at a density of $1 \times 104 /$ well, and cultured in a DMEM/F12 medium containing $10 \%$ fetal bovine serum at $37{ }^{\circ} \mathrm{C}$ in a $5 \% \mathrm{CO}_{2}$ incubator. Each group included 5 duplicate holes. $50 \mu \mathrm{L}$ MTT solution was added at 24,48 , and $72 \mathrm{~h}$ after transfection, and the culture was continued for $4 \mathrm{~h}$. After discarding the supernatant, $200 \mu \mathrm{L}$ DMSO was added. The A value was measured at $490 \mathrm{~nm}$.

\section{Flow cytometry}

$1 \times 10^{6}$ cells were washed twice with PBS. Then $200 \mu \mathrm{L}$ binding buffer and $10 \mu \mathrm{L}$ FITC-labeled Annexin-V $(20 \mu \mathrm{g} / \mathrm{mL})$ and $5 \mu \mathrm{L}$ PI $(50 \mu \mathrm{g} / \mathrm{mL})$ were added, and the cells were incubated in dark at room temperature for $30 \mathrm{~min}$. Then $400 \mu \mathrm{L}$ PBS was added, and cells were analyzed by using FACScalibur cytometer and Cell-Quest Version 3.2.1 software. A tube without Annexin V-FITC and PI was used as a negative control. All experiments are repeated three times.

\section{Dual luciferase assay}

To construct MALAT1-WT, MALAT1-MUT, AKT3WT, AKT3-MUT luciferase reporters, 3'UTR of MALAT1-WT, MALAT1-MUT, AKT3-WT, AKT3MUT was amplified and inserted into the pmirGLO vector (Invitrogen, Carlsbad, CA, USA). MALAT1-WT, MALAT1-MUT, AKT3-WT or AKT3-MUT and miR150-5p, miR-NC, anti-NC or anti-miR-150-5p were cotransfected into the chondrocytes using Lipofectamine 2000 (ThermoFisher Scientific). $48 \mathrm{~h}$ after transfection, dual-luciferase reporter gene assays were performed to measure the luciferase activity with Dual-Luciferase Reporter Assay System kit (Promega, USA).

\section{Anti-Ago2 RIP assay}

Chondrocytes cells were transfected with miR-NC or miR-150-5p respectively. $48 \mathrm{~h}$ after transfection, RIP assays were performed with the transfected cells by using the Magna RIPTM RNA Binding Protein Immunoprecipitation Kit (Millipore, Bedford, MA, USA). Then the cells were incubated with anti-Ago2 antibody (Millipore) or negative control IgG (Millipore), and the relative enrichment of MALAT1 and AKT3 were measured by qRT-PCR.

\section{Statistical analysis}

Data were shown as the mean $\pm S D$. Prism software was used to analysis data. Data between two groups were analyzed by using the t-test. Data from more than two groups were analyzed using the One-Way ANOVA method. All experiments were performed and analyzed in triplicate. $\mathrm{P}<0.05$ was considered significant.

\section{Results}

Different expression levels of MALAT1, miR-150-5p and $A K T 3$ in normal and $O A$ cartilage tissues

We performed qRT-PCR to examine the expressions of MALAT1, miR-150-5p and AKT3 in OA and normal cartilage tissues respectively. As shown in Fig. 1a the expressions of MALAT1 and AKT3 were significantly upregulated in OA cartilage tissues compared with that of normal tissues. On the contrary, the expression of miR$150-5 p$ was markedly reduced in OA cartilage tissues. The western blot analysis of AKT3 was also consistent with the qRT-PCR result which displayed an increased level of AKT3 in OA cartilage tissues (Fig. 1b). In addition, to evaluate the relationship between MALAT1, miR-150-5p and AKT3, we used Spearman to analyze the correlations between them. As displayed in Fig. 1c, the expression level of miR-150-5p was negatively correlated with that of MALAT1 or AKT3. Furthermore, the chondrocytes treated by $10 \mathrm{ng} / \mathrm{mL} \mathrm{IL}-1 \beta$ for $4,6,12$, and $24 \mathrm{~h}$ showed the increased expression of MALAT1 and AKT3 while decreased expression of miR-150-5p with the increase of treatment in time, consistent with the result in OA cartilage tissues (Fig. 1d, e). In the following studies, OA progress was induced by the treatment of chondrocytes with $10 \mathrm{ng} / \mathrm{mL}$ IL-1 $\beta$. These results indicated that the expressions of MALAT1 and AKT3 were both up-regulated in OA, while the expression of miR-150-5p was down-regulated.

\section{MALAT1 indirectly regulated AKT3 through targeting miR-150-5p}

It had been found in previous studies that MALAT1 could achieve its function through correlating with miRNAs in OA progression. For further exploration of the potential mechanism of MALAT1 in OA progression, we firstly searched for the miRNAs correlated with MALAT1 by miRcode. Then, the potential miRNA binding sites in MALAT1 were predicted and miR-150-5p was selected to be the possible target of MALAT1 (Fig. 2a). Besides, the luciferase reporter assay was carried out to confirm the relationship between MALAT1 and miR-150-5p. As shown in 

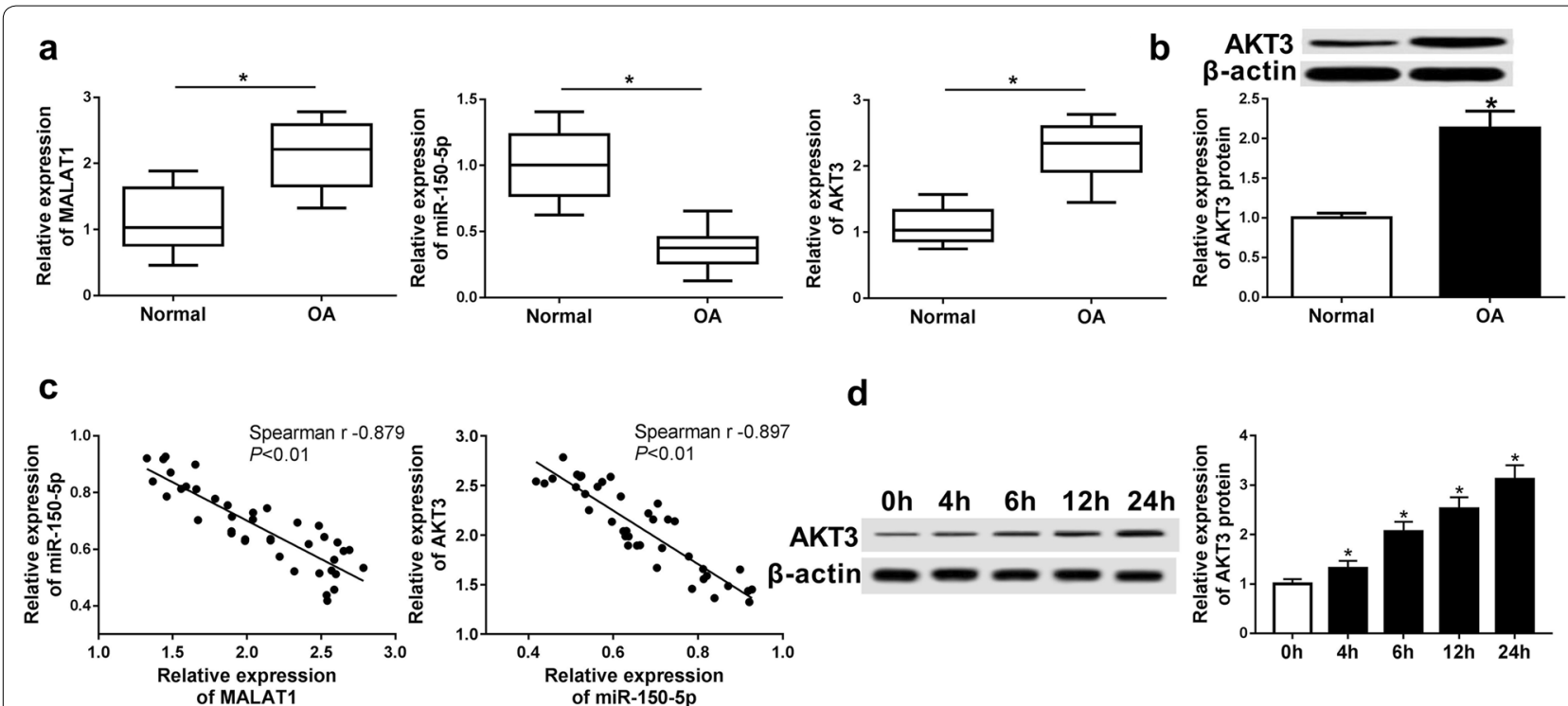

d
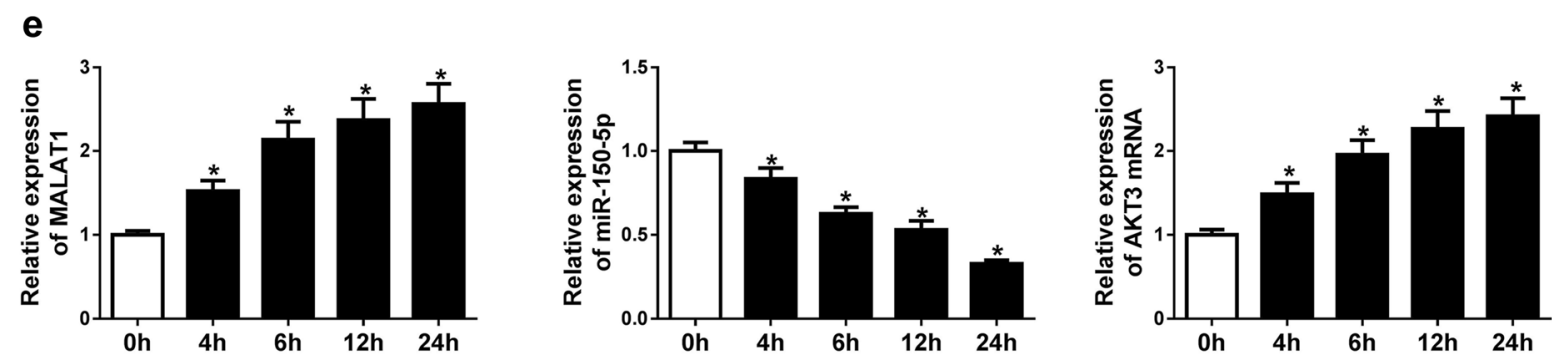

Fig. $\mathbf{1}$ Different expression levels of MALAT1, miR-150-5p and AKT3 in normal and OA cartilage chondrocytes. $\mathbf{a}$, b The expression levels of MALAT1, miR-150-5p and AKT3 in OA and normal cartilage tissues were detected by qRT-PCR and western blot. Samples were from 42 OA patients and 20 healthy subscribers. c Spearman was used to analyze the relationship between MALAT1, miR-150-5p and AKT3. d, e qRT-PCR and western blot were used to examine the levels of MALAT1, miR-150-5p and AKT3 in chondrocytes with treatment of $10 \mathrm{ng} / \mathrm{mL} \mathrm{IL}-1 \beta$ for $4,6,12$, and $24 \mathrm{~h}$. ${ }^{*} P<0.05$

Fig. 2b, MALAT1 was directly targeted to miR-150-5p. In addition, we took anti-Ago2 RIP assay to illuminate the endogenous relationship between MALAT1 and miR-150-5p. As suggested in Fig. 2c, MALAT1 were specifically enriched in the Ago2 pellet compared with control IgG immunoprecipitates. Moreover, we performed bioinformatics analyses (TargetScan, miroRNA. org and Starbase v2.0) to search for the potential target mRNAs of miR-150-5p and found AKT3 as a potential target (Fig. 2d). Dual luciferase reporter assays (Fig. 2e) and anti-Ago2 RIP assay (Fig. 2f) were also performed to identify the interaction of miR-150-5p and AKT3, and the results proved that AKT3 was a direct target of miR-150-5p. Thus, we thought that MALAT1 may achieve its function by directly targeting miR-150-5p, which targets AKT3 to produce influence on chondrocytes in OA.

\section{MALAT1 indirectly promotes AKT3 expression by competitively binding to miR-150-5p}

In order to further explore the regulation of MALAT1 on AKT3, MALAT1 and miR-150-5p were up-regulated or down-regulated in chondrocytes before IL-1 $\beta$ treatment. The expression of AKT3 was measured by qRT-PCR and western blot. The transfection efficiency revealed that MALAT1 was upregulated in IL-1 $\beta$-stimulated chondrocytes after transfected with MALAT1 overexpression plasmid (Fig. 3a). Similarly, the level of miR-150-5p was elevated using miR-150-5p mimic while it was decreased using anti-miR-150-5p transfection (Fig. 3b, c) in IL- $1 \beta$-stimulated chondrocytes. AKT3 protein expression level was much higher when MALAT1 was up-regulated (Fig. 3d), On the contrary, the down-regulation of MALAT1 led to a decreased expression level of AKT3 (Fig. 3e). The overexpression of miR-150-5p decreased AKT3 expression level, while the expression of AKT3 was notably increased after transfecting with anti-miR-150-5p 
a

\begin{tabular}{ll}
\hline MALAT1-WT & 5'-AAAUGGAAAGAUUAAUUGGGAGU-3' \\
miR-150-5p & 3'-GUGACCAUGUU---CCCAACCCUCU- 5' \\
MALAT1-MUT & 5'- AAAUGGAAAGAUUAAGGUUACGU-3'
\end{tabular}

b

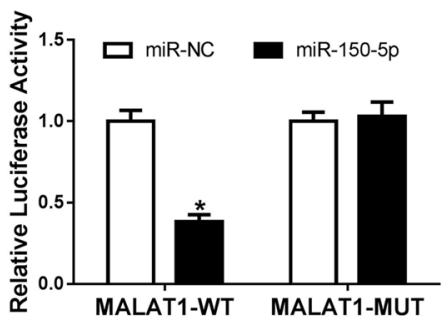

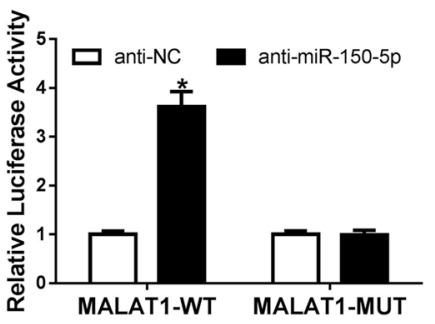

C

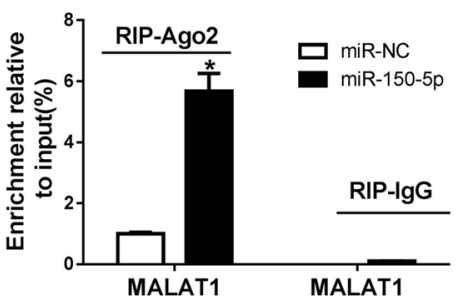

d

\begin{tabular}{ll}
\hline AKT3-WT & 5'- GUUAGAAUUUGGCUUUGGGAGA-3' \\
miR-150-5p & 3'-GUGACCAUGUU---CCCAACCCUCU- 5' \\
AKT3-MUT & 5'- GUUAGAAUUUGGCUGGCCAUGA-3'
\end{tabular}

e

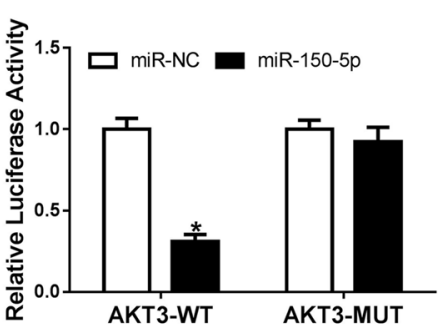

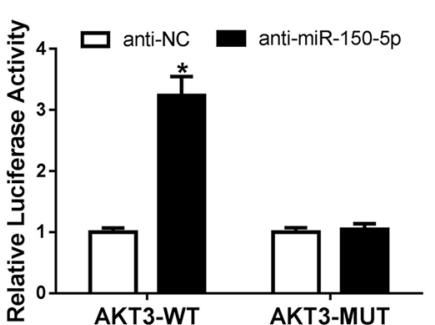

f

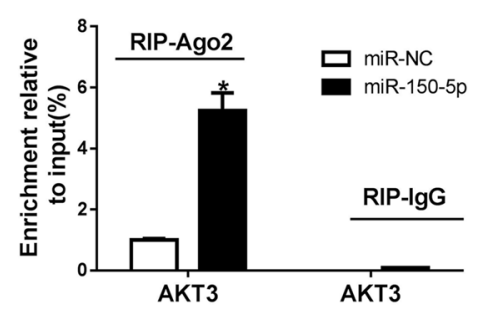

Fig. 2 MALAT1 indirectly regulates AKT3 by targeting miR-150-5p. a The surmised binding sites for interaction between miR-150-5p and MALAT1. b Dual luciferase reporter assay was performed to conform the interaction between MALAT1 and miR-150-5p. c Anti-Ago2 RIP assay was carried out to identify the correlation between MALAT1 and miR-150-5p. d The surmised binding sites for correlation between miR-150-5p and AKT3. e Dual luciferase reporter assay was performed to confirm the interaction between miR-150-5p and AKT3. f Anti-Ago2 RIP assay was carried out to identify the correlation between miR-150-5p and AKT3. ${ }^{*} P<0.05$

(Fig. 3f, g). After MALAT1 and miR-150-5p were cotransfected into chondrocytes, the MALAT1 overexpression-mediated promotion of AKT3 was hampered by miR-150-5p mimic transfection (Fig. 3h). After cotransfecting chondrocytes with si-MALAT1 and antimiR-150-5p, the suppression of AKT3 by si-MALAT1 was rescued by anti-miR-150-5p (Fig. 3i). These observations demonstrated that MALAT1 may act as a competing endogenous RNA (ceRNA) and could indirectly promote the expression of AKT3 through competitively binding to miR-150-5p.
MALAT1 knockdown suppresses cell proliferation while induces apoptosis during $\mathrm{OA}$ progression We treated chondrocytes with $10 \mathrm{ng} / \mathrm{mL}$ IL- $1 \beta$ for $24 \mathrm{~h}$ after transfection to investigate the influence of MALAT1 knockdown on cell apoptosis and proliferation in OA. The interfering RNA for MALAT1 was introduced into chondrocytes before IL-1 $\beta$ treatment. The expression of MALAT1 was significantly downregulated in IL-1 $\beta+$ si-MALAT1 group compared with that of $\mathrm{IL}-1 \beta+\mathrm{si}-\mathrm{NC}$ group (Fig. 4a). MTT results 

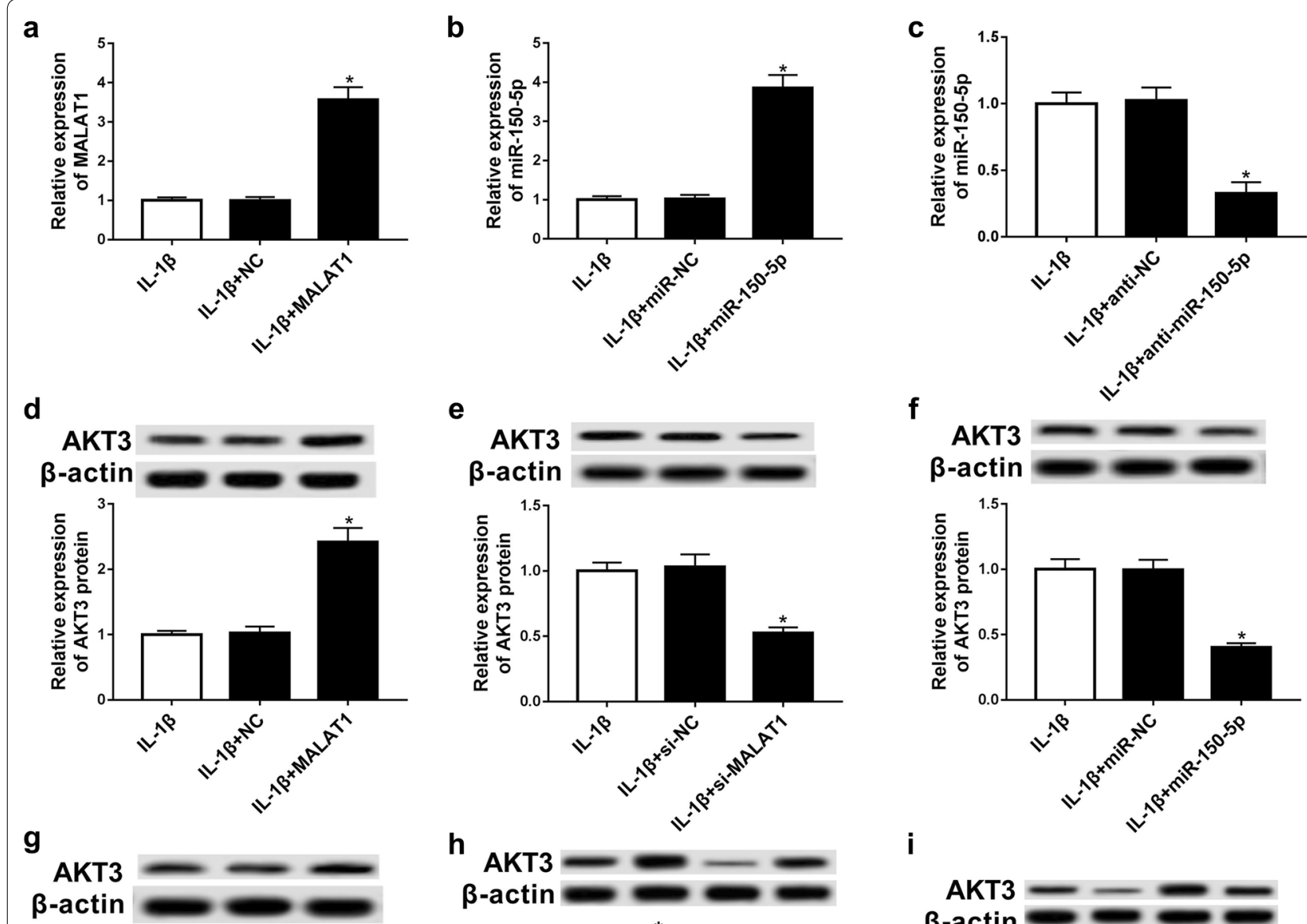

\section{h}

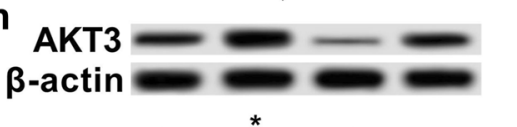

i
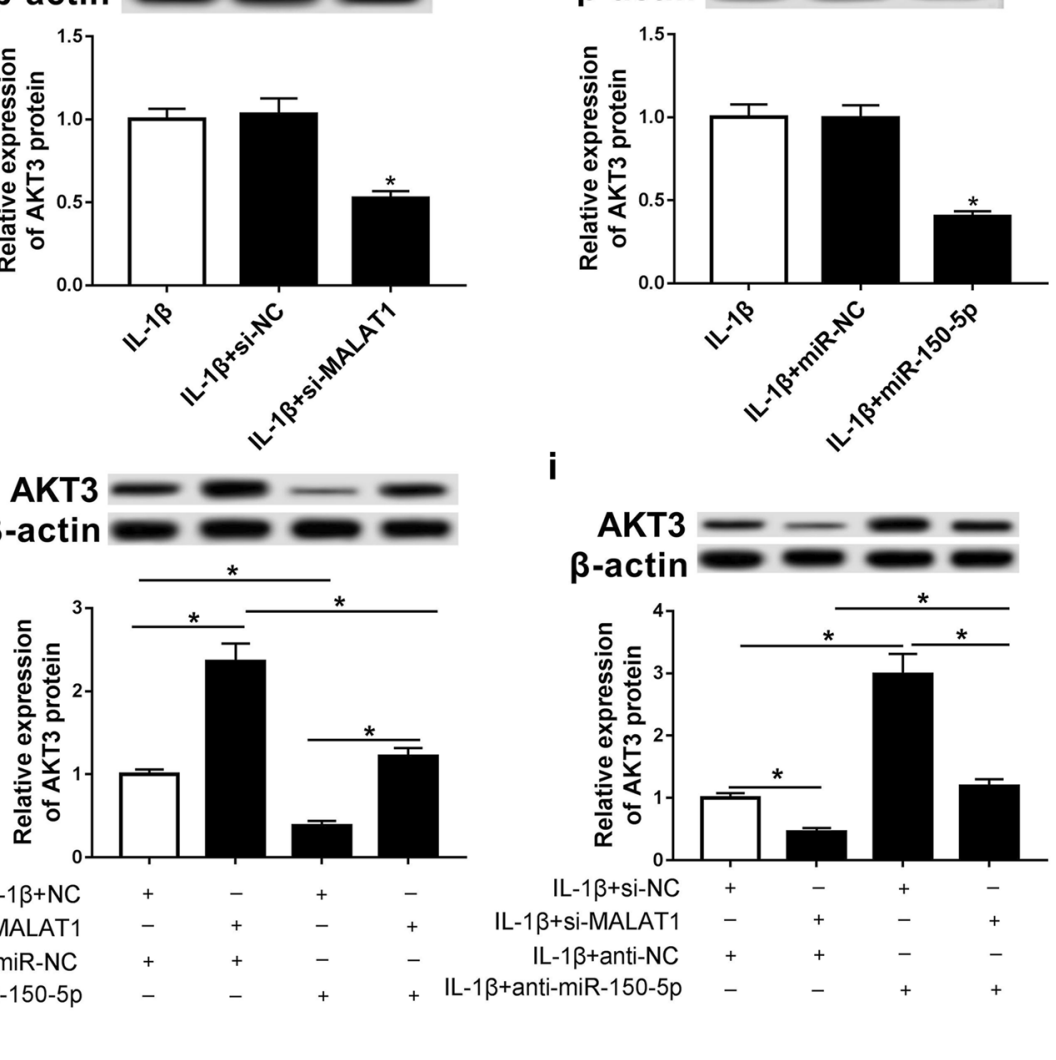

IL-1 $\beta+$ Si-NC

IL-1 $\beta+$ Si-MALAT1

IL-1 $\beta+a n t i-N C$

IL-1 $\beta+m i R-N C$

IL-1 $\beta+m i R-150-5 p$

Fig. 3 MALAT1 competitively bound to microRNA-150-5p and indirectly promotes AKT3 expression. a-c Chondrocytes were introduced with miR-150-5 $p$ mimic, miR-NC, anti-miR-150-5p, anti-miR-NC, NC, or MALAT1 before IL-1 $\beta$ treatment. qRT-PCR was used to measure the level of miR-150-5p. $\mathbf{d}$-i Western blot analysis of AKT3 in each group. ${ }^{*} \mathrm{P}<0.05$

demonstrated that cell proliferation was reduced in MALAT1 knockdown chondrocytes (Fig. 4b). Besides, the flow cytometry results indicated that the apoptosis of chondrocytes was increased after MALAT1 knockdown (Fig. 4c, d). Moreover, the apoptosis-related proteins cleaved-PARP, Bax, and Bcl-2 were also detected. We disclosed that anti-apoptosis-related protein
Bcl-2 was downregulated, while pro-apoptosis-related protein cleaved-PARP and Bax were upregulated in IL-1 $\beta+$ si-MALAT1 group compared with that of IL-1 $\beta+$ si-NC group (Fig. 4e). These findings suggested that MALAT1 might affect the process of OA by regulating the proliferation and apoptosis of chondrocytes. 


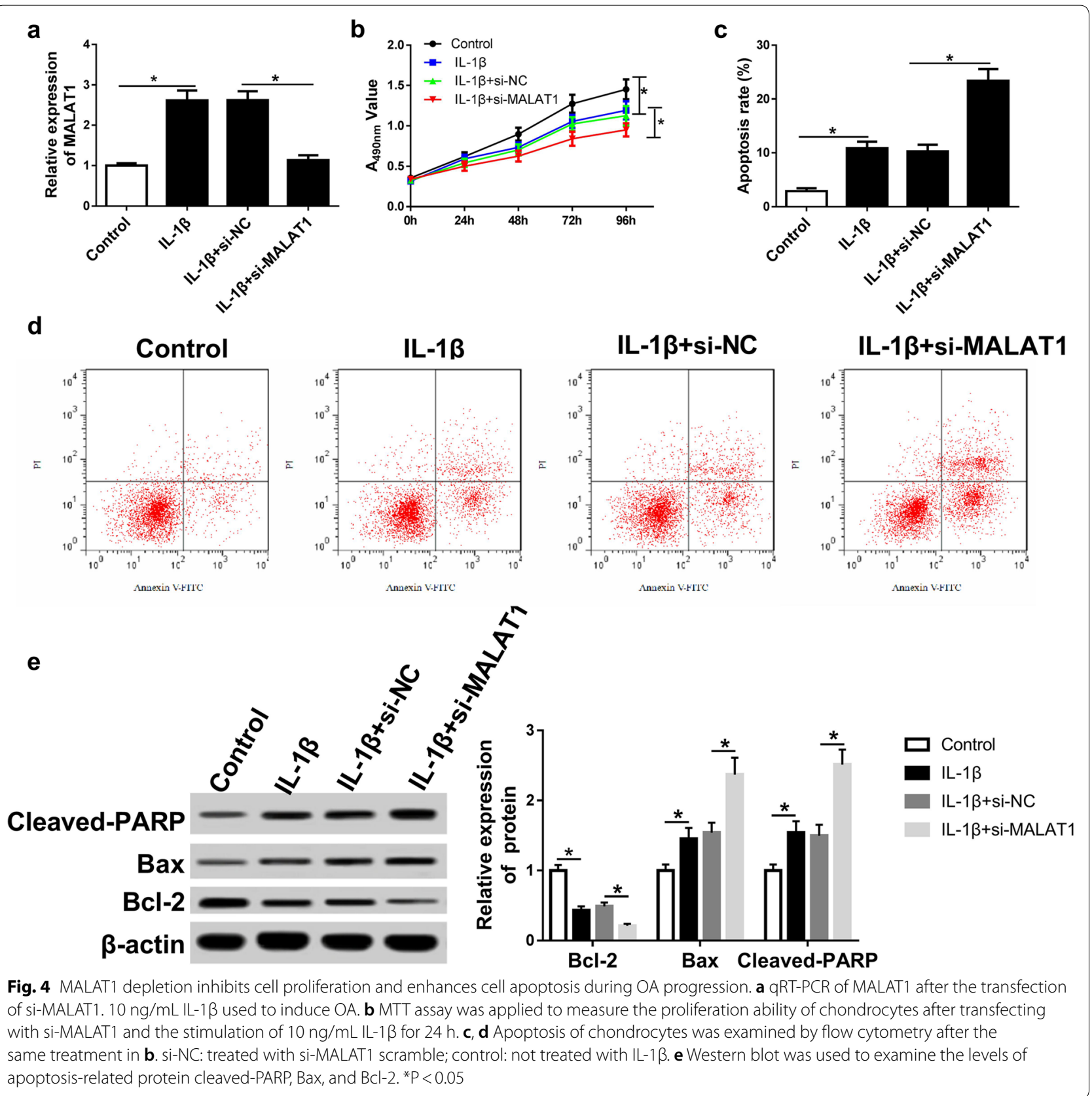

MALAT1 depletion promotes the ECM degradation of OA chondrocytes

To further investigate the effect of MALAT1 on IL-1 $\beta$ induced ECM degradation in chondrocytes, we mainly detected four critical cartilage-related genes: MMP-13, ADAMTS-5, collagen II and aggrecan. MMP-13 and ADAMTS-5 were known as major cartilage-degrading enzymes, and collagen II and aggrecan were identified as vital extracellular matrix (ECM) proteins in cartilage tissues. The western blot results in Fig. 5 displayed that the expression of MMP-13 and ADAMTS-5 were largely increased after transfecting with si-MALAT1 in OA chondrocytes. Contrarily, the protein expression levels of collagen II and aggrecan were significantly lower relative to si-NC control (Fig. 5). Taken together, these results demonstrated that MALAT1 depletion may up-regulate cartilage-degrading enzymes while down-regulate extracellular matrix proteins, thus promoting the ECM degradation of OA chondrocytes. 

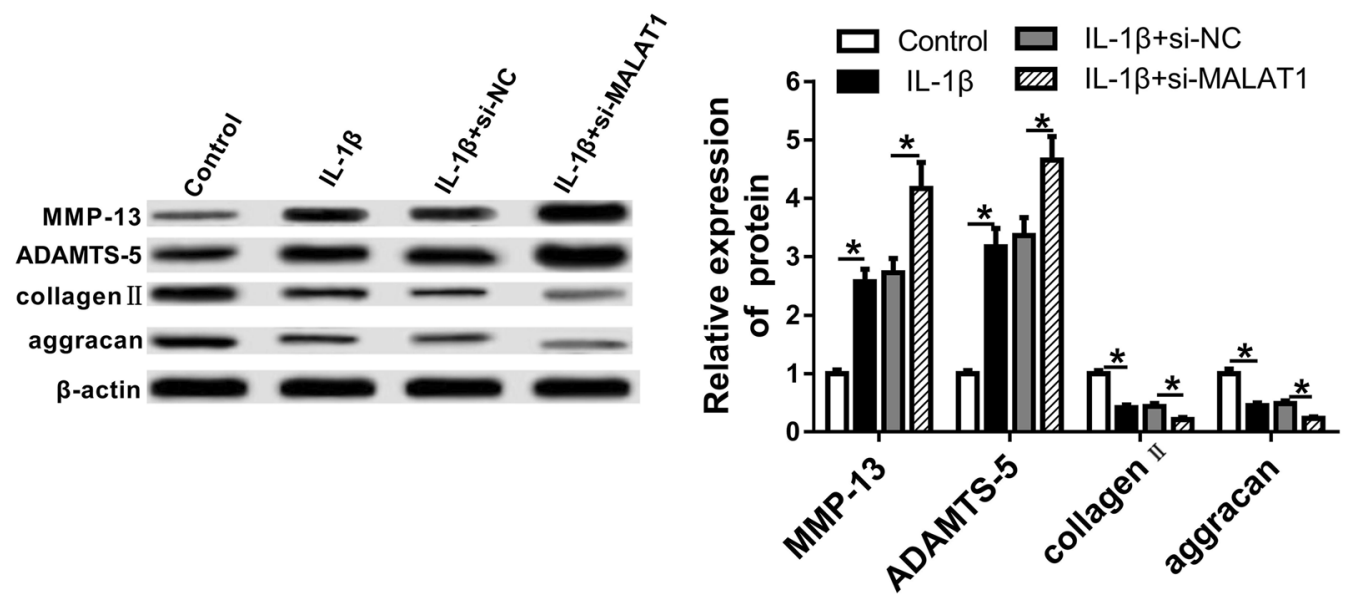

Fig. 5 MALAT1 knockdown promotes the ECM degradation of chondrocytes in OA. The protein expression levels of MMP-13, ADAMTS-5, collagen $\|$ and aggrecan detected by western blot after transfecting with si-MALAT1 and stimulating with IL-1 $\beta$ (10 ng/mL) for $24 \mathrm{~h}$. $\beta$-actin was used as an internal control. si-NC: treated with si-MALAT1 scramble; Control: not treated with IL-1 $\beta$. ${ }^{*} P<0.05$

\section{MiR-150-5p reverses the influences of MALAT1 on proliferation and apoptosis in IL-1 $\beta$-induced chondrocytes}

The rescue experiments were performed to further investigate whether MALAT1 regulates cell proliferation cell apoptosis via miR-150-5p in OA. As shown in Fig. 6a, the transfection efficiency demonstrated that AKT3 overexpression plasmid or si-AKT3 could significantly induce the increase or decrease of AKT3, respectively. MTT assay was conducted to determine the proliferation ability of chondrocytes, the results shown in Fig. 6b demonstrated that up-regulation of MALAT1 or AKT3 strikingly promoted the proliferation of chondrocytes. However, this effect was dramatically blocked when miR150-5p was overexpressed. Oppositely, cell proliferation ability was evidently suppressed in MALAT1 or AKT3 down-regulated $\mathrm{OA}$ chondrocytes, while the inhibition of miR-150-5p markedly reversed the effect. The results of flow cytometry were shown in Fig. 6c, overexpression of MALAT1 or AKT3 significantly inhibited the chondrocytes apoptosis while the influence was reversed by miR-150-5p overexpression. On the contrary, cell apoptosis was increased in MALAT1 or AKT3 knockdown OA chondrocytes, while the inhibition of miR-150-5p reversed the effect evidently. These results indicated that MALAT1 probably promoted proliferation and inhibited apoptosis in OA chondrocytes by competitively binding to miR-150-5p and indirectly regulating AKT3.

\section{MiR-150-5p reverses the effects of MALAT1 on ECM degradation of IL-1 $\beta$-induced chondrocytes}

The rescue experiments were performed to further explore whether MALAT1 suppresses ECM degradation and facilitates cartilage formation through miR-150-5p/ AKT3 axis in OA. MMP-13, ADAMTS-5, collagen II and aggrecan were detected by using western blot. When OA chondrocytes were transfected with MALAT1 or AKT3 overexpression plasmids, the protein expression levels of MMP-13 and ADAMTS-5 were decreased while the levels of collagen II and aggrecan were evidently increased. However, these effects were blocked by the overexpression of miR-150-5p (Fig. 7a). Contrarily, in MALAT1 or AKT3 knockdown OA chondrocytes, the expression levels of MMP-13 and ADAMTS-5 were significantly increased and the levels of collagen II and aggrecan were markedly reduced, while the inhibition of miR-150-5p dramatically reversed the effects (Fig. 7b). These findings demonstrated MALAT1 might inhibits ECM degradation and promotes cartilage formation via miR-150-5p/AKT3 axis in $\mathrm{OA}$.

\section{Discussion}

LncRNA MALAT1, an important lncRNA, is expressed in a variety of tissues and involved in a variety of diseases and biological processes [29]. In various cancers, the expression of MALAT1 is usually upregulated. The up-regulation of MALAT1 can enhance proliferation and inhibit apoptosis, and the down-regulation can reduce proliferation and promote apoptosis. MALAT1 expression level is significantly up-regulated in glioma stem cells, this up-regulation enhances the viability and proliferation of glioma stem cells and promotes the occurrence of glioma tumors [30]. The plasma level of MALAT1 is increased in patients with early fever after breast cancer surgery, but the inflammatory responses and metastasis of lung are decreased significantly after 


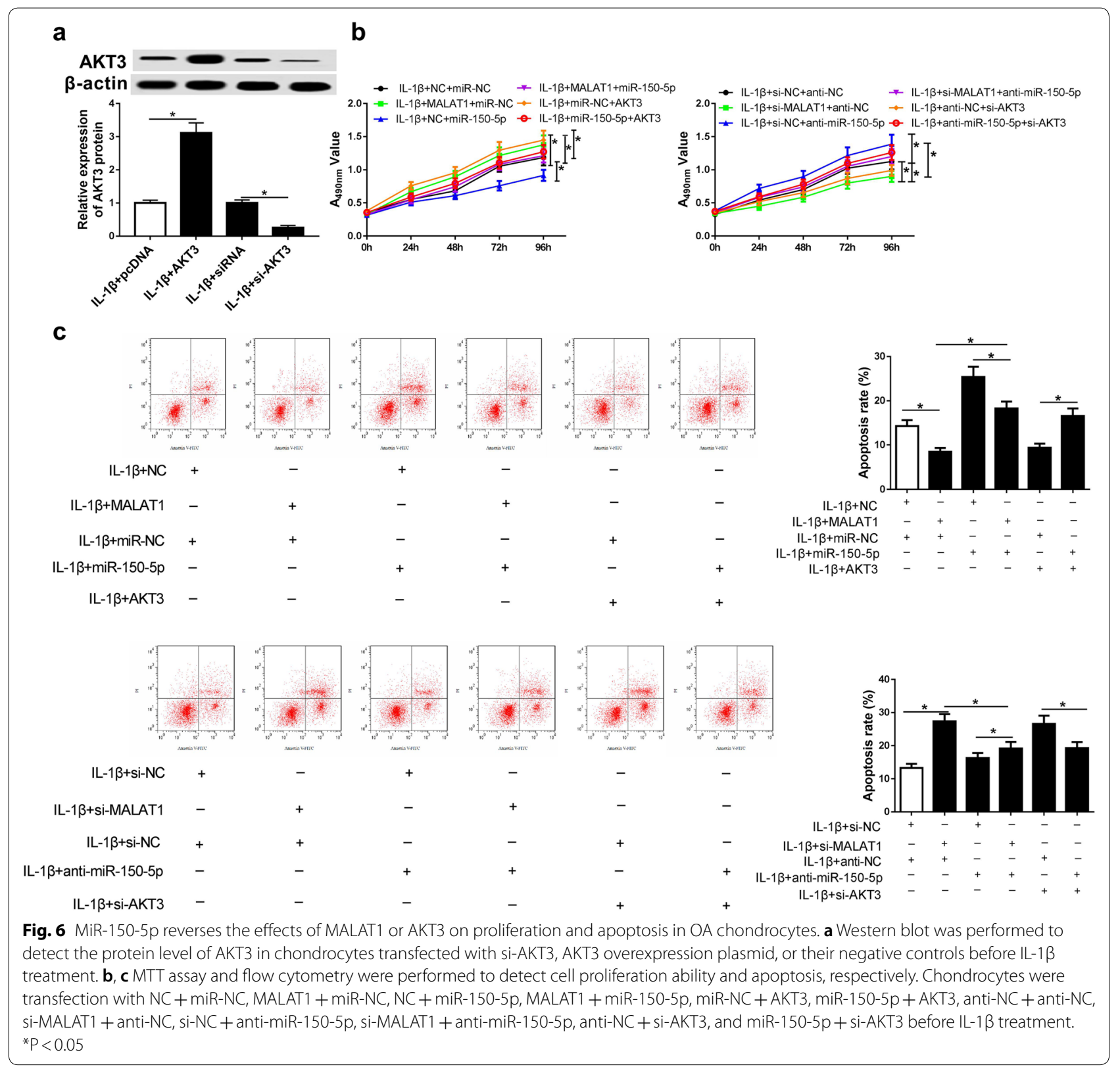

down-regulation of MALAT1 [31]. MALAT1 is up-regulated in human colon cancer cell lines and inhibition of MALAT1 could reduce the proliferation of colon cancer cells [32]. In another report, MALAT1 downregulation markedly represses human OA chondrocyte proliferation [33]. Consistent with previous studies, our study showed that the expression of MALAT1 was up-regulated in OA chondrocytes compared with normal chondrocytes and has a positive correlation with the severity of OA. MALAT1 knockdown in IL-1 $\beta$ induced chondrocytes inhibited cell proliferation while promoted cell apoptosis. Thus, we predicted that MALAT1 may have an important function in contributing to proliferation ability and restraining apoptosis in OA progression, thus promoting OA process.

Extracellular matrix ECM degradation and ECM production are two important factors affecting chondrocyte destruction [5, 34]. Members of the MMP and ADAMTS gene family play important roles in ECM degradation. Aggrecan and type II collagen are considered largely contributed to the formation of cartilage tissue $[4,35]$. Our experiment measured the degradation and formation of ECM by western blot detection of MMP-13, ADAMTS-5, aggrecan and type II collagen. The down-regulation of 

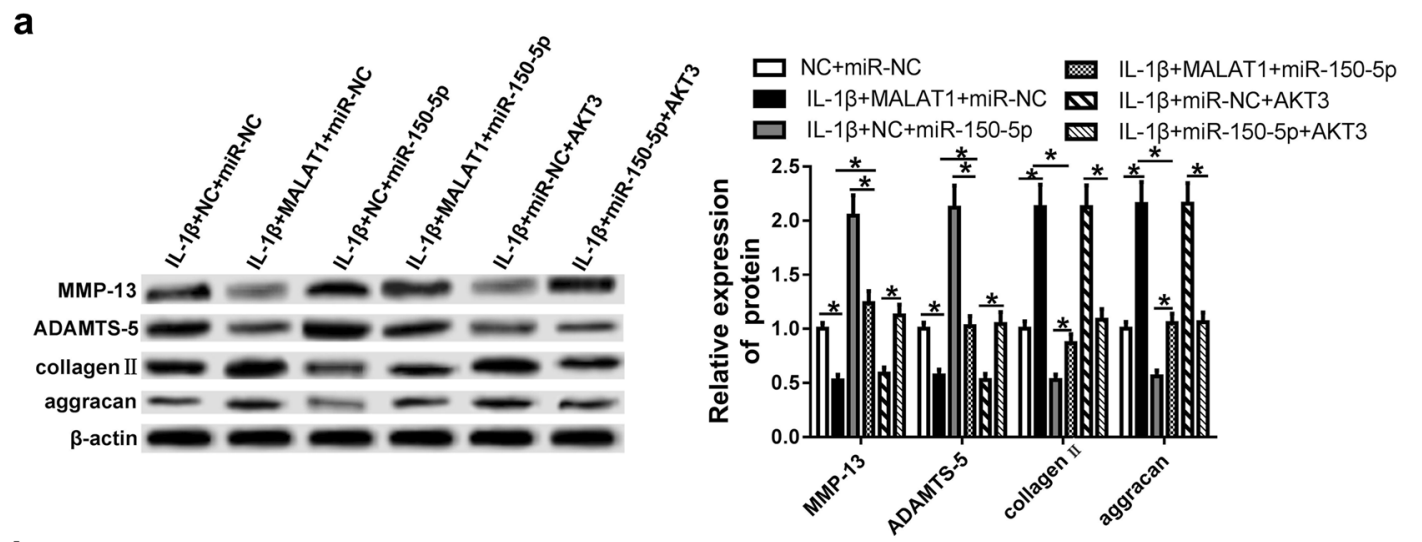

b

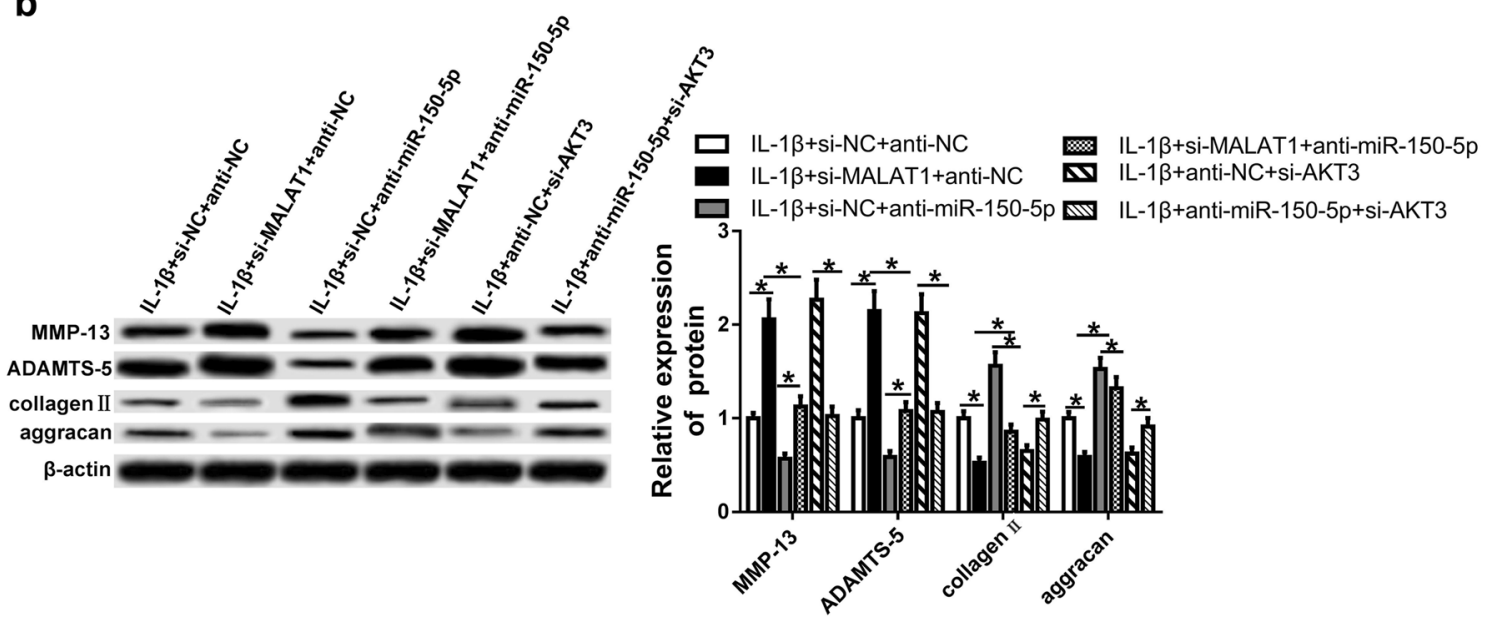

Fig. 7 MiR-150-5p reverses the effects of MALAT1 or AKT3 on ECM degradation in OA. $\mathbf{a}$, b Western blot of MMP-13, ADAMTS-5, collagen II and aggrecan after transfection with NC + miR-NC, MALAT1 + miR-NC, NC + miR-150-5p, MALAT1 + miR-150-5p, miR-NC + AKT3, miR-150-5p + AKT3, anti-NC + anti-NC, si-MALAT1 + anti-NC, si-NC + anti-miR-150-5p, si-MALAT1 + anti-miR-150-5p, anti-NC + si-AKT3, and miR-150-5p + si-AKT3 before IL-1 $\beta$ treatment. ${ }^{*} \mathrm{P}<0.05$

MALAT1 increased MMP-13 and ADAMTS-5 while decreased collagen II and aggrecan in OA. Those data indicated that depletion of MALAT1 may contribute to ECM degradation and play a key role in the pathogenesis of OA. Previous studies have reported that MALAT1 could achieve its function through correlating with miRNAs [36-38]. For example, Lei et al. reported that MALAT1 participates in the ovarian cancer growth by sponging miR-506 [36]. Chang et al. also disclosed that MALAT1 could act as a ceRNA to regulate signal transducer and activator of transcription-3 (STAT3) in oral squamous cell carcinoma [37]. In this study, we found that miR-150-5p may be act as the possible target of MALAT1 by miRcode. Subsequently, we further disclosed that AKT3 was a direct target of miR-150-5p. Moreover, MALAT1 increased the expression of AKT3 by sponging miR-150-5p. Interestingly, A low expression of miR-150-5p and high expression of AKT3 were also observed in OA cartilage tissue. Therefore, we thought that MALAT1 may play important roles in OA progression by miR-150-5p/AKT3.

A series studies have found that AKT was important in growth, proliferation and metabolism and MALAT1 might influenced the proliferation and metastasis of cells by regulating AKT [39-44]. Dong et al. revealed that MALAT1 could inhibit proliferation and invasion through PI3K/AKT signaling pathway [39]. AKT3 inhibition has been reported to contribute to cell apoptosis in embryonic stem cells [45]. Researchers have found that AKT3 is elevated in OA chondrocytes [46]. But, the involvement of AKT3 in MALAT1-mediated function in OA remain largely unknown. In addition, many studies have demonstrated that miR-150-5p could suppress tumor cell proliferation and migration, facility tumor cell apoptosis, and inhibit the process of tumor [47-49]. For instance, miR-150-5p curbs cell proliferation, migration, invasion and angiogenesis in colorectal cancer [48]. Recently, miR-150-5p was found to be downregulated in 


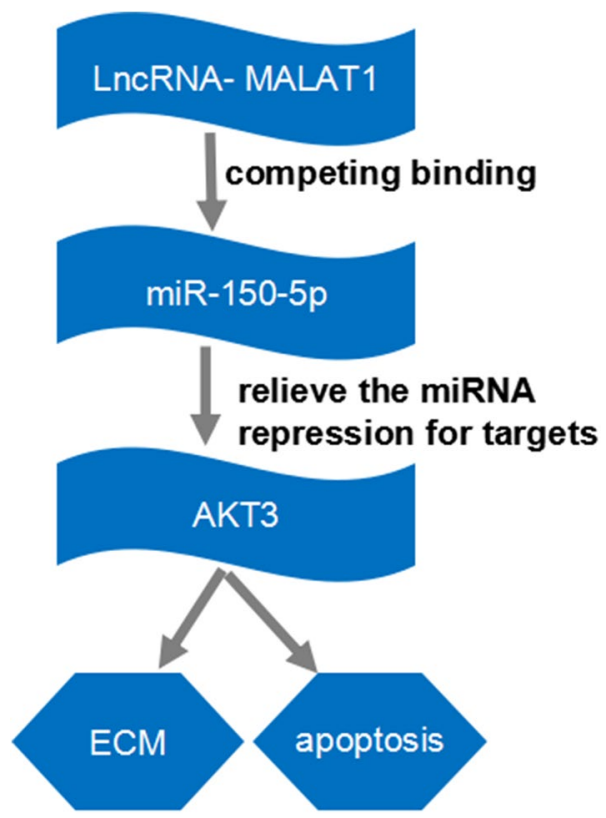

Fig. 8 The schematic diagram of in MALAT1/MiR-150-5p/AKT3OA axis in OA. Lnc RNA MALAT1 could be act as a ceRNA of miR-150-5p to regulate the expression of AKT3, thus modulating the ECM degradation and cell apoptosis

OA patients [50]. Yang et al. further disclosed that miR$150-5 p$ is elevated in Interleukin-1-mediated human chondrogenic cells ATDC5 and loss of miR-150 could alleviate IL-1-induced cell damage in ATDC5 cells [51]. However, whether miR-150-5p is involved in the function of MALAT1 in OA has not been reported. In this report, we demonstrated that overexpression of MALAT1 inhibited cell apoptosis while enhanced proliferation, which was rescued by upregulation of miR-150-5p. Similarly, the depletion of miR-150-5p also attenuated the effect of absence of MALAT1. More importantly, the AKT3 also weakened the effect of miR-150-5p on cell proliferation and apoptosis. Those data suggested that MALAT1 may modulate the cell proliferation and apoptosis by miR150-5p/AKT3 axis. Further rescue experiments found that miR-150-5p reversed the influences of MALAT1 on ECM degradation of IL- $1 \beta$-induced chondrocytes. Besides, AKT3 also undermined the effect of miR-150-5p in IL-1 $\beta$-induced chondrocytes, further suggesting that MALAT1 may be responsible for the cell proliferation, apoptosis and ECM degradation by miR-150-5p/AKT3 axis (Fig. 8).

\section{Conclusion}

Taken together, we provide the evidence that MALAT1 contributed to OA progression by promoting cell proliferation and cartilage formation, and inhibiting cell apoptosis and ECM degradation. And the underlying regulatory mechanism is that MALAT1 exited its function through acting as a sponge for miR-150-5p and indirectly regulating AKT3, thus forming a MALAT1/miR-150-5p/ AKT3 axis to participate in OA progression. The regulatory mechanism indicated that MALAT1 might be used as a new drug target for the treatment of OA.

\section{Abbreviations \\ OA: osteoarthritis; IncRNAs: long non-coding RNAs; miRNAs: microRNAs; qRT- PCR: quantitative real-time PCR; PVDF: polyvinylidene difluoride.}

\section{Acknowledgements}

We would like to take this opportunity to express our sincere gratitude to the National Nature Science Foundation of China and Southwest Hospital, the Third Military Medical University for their strong support to this research.

\section{Funding}

This study was supported by: The National Nature Science Foundation of China (Grant No. 81301594). Third Military Medical University, Southwest Hospital Fund (Grant No. SWH2016LCYB-17)

\section{Availability of data and materials}

Not applicable.

\section{Ethics approval and consent to participate}

This study was approved by the Research Ethics Committee of Southwest Hospital, the Third Military Medical University (Army Medical University). And all the donors and their families read and signed the informed consents.

\section{Consent for publication}

Not applicable.

\section{Competing interests}

The authors declare that they have no competing interests.

Received: 22 January 2019 Accepted: 7 May 2019

Published online: 01 July 2019

\section{References}

1. Berenbaum F. Osteoarthritis is an inflammatory disease. Osteoarthritis Cartilage. 2012;20:S5

2. Arden N, Nevitt MC. Osteoarthritis: epidemiology. Best Prac Res Clin Rheumatol. 2006:20:3-25.

3. Wieland HA, Michaelis M, Kirschbaum BJ, Rudolphi KA. Osteoarthritis-an untreatable disease? Nat Rev Drug Discov. 2005;4:331-44.

4. Hu J, Wang Z, Shan Y, Pan Y, Ma J, Jia L. Long non-coding RNA HOTAIR promotes osteoarthritis progression via miR-17-5p/FUT2/ $\beta$-catenin axis. Cell Death Dis. 2018;9:711.

5. Okada A, Okada Y. Progress of research in osteoarthritis. Metalloproteinases in osteoarthritis. Clin Calcium. 2009;19:1593-601.

6. Zhang W, Nuki G, Moskowitz RW, Abramson S, Altman RD, Arden NK, Bierma-Zeinstra S, Brandt KD, Croft P, Doherty M. OARSI recommendations for the management of hip and knee osteoarthritis: part III: changes in evidence following systematic cumulative update of research published through January 2009. Osteoarthritis Cartilage. 2010;18:476-99.

7. Majeed MH, Sherazi SAA, Bacon D, Bajwa ZH. Pharmacological treatment of pain in osteoarthritis: a descriptive review. Curr Rheumatol Rep. 2018;20:88.

8. Wollheim FA. Current pharmacological treatment of osteoarthritis. Drugs. 1996;52(Suppl 3):27-38.

9. Zhang S, Guan X. Latest development of external treatment with traditional Chinese medicine for knee osteoarthritis. Chin J Osteoporosis. 2016;22:907-11. 
10. John R, David H, James I, Morgan HJ, Lynn S-M, Daniel VD, Cheryl R, Elizabeth GM, Robert GM, Bruce AL. American Academy of Orthopaedic Surgeons clinical practice guideline on the treatment of osteoarthritis (OA) of the knee. J Bone Joint Surg. 2010;92:990-3.

11. Brosseau L, Wells G, Marchand S, Gaboury I, Stokes B, Morin M, Casimiro L, Yonge K, Tugwell P. Randomized controlled trial on low level laser therapy (LLLT) in the treatment of osteoarthritis (OA) of the hand. Lasers Surg Med. 2010;36:210-9.

12. Karsdal MA, Tanko LB, Riis BJ, Sondergard BC, Henriksen K, Altman RD, Qvist P, Christiansen C. Calcitonin is involved in cartilage homeostasis: is calcitonin a treatment for OA? Osteoarthritis Cartilage. 2006;14:617-24.

13. Baker CL, Ferguson CM. Future treatment of osteoarthritis. Orthopedics. 2005;28:S227-34.

14. Liu Z, Cao A, Jiang L, Cao S. Biological function and regulatory mechanism of long non-coding RNA (IncRNA). J Agric Biotechnol. 2018;26:1419-30.

15. Li J, Meng H, Bai Y, Wang K. Regulation of IncRNA and its role in cancer metastasis. Oncol Res. 2016;23:205-17.

16. Zhu JJ, Fu HJ, Wu YG, Zheng XF. Function of IncRNAs and approaches to IncRNA-protein interactions. Sci China-Life Sci. 2013;56:876-85.

17. Orly W, Howard YC. Long noncoding RNAs and human disease. Trends Cell Biol. 2011;21:354-61.

18. Cao L, Wang Y, Wang Q, Huang J. LncRNA FOXD2-AS1 regulates chondrocyte proliferation in osteoarthritis by acting as a sponge of miR-206 to modulate CCND1 expression. Biomed Pharmacother. 2018;106:1220-6.

19. Li Y, Li S, Luo Y, Liu Y, Yu N. LncRNA PVT1 regulates chondrocyte apoptosis in osteoarthritis by acting as a sponge for miR-488-3p. DNA Cell Biology. 2017;36:571-80.

20. Shen H, Wang Y, Shi W, Sun G, Hong L, Zhang Y. LncRNA SNHG5/miR-26a/ SOX2 signal axis enhances proliferation of chondrocyte in osteoarthritis. Acta Biochim Biophys Sin. 2018;50:191-8.

21. Wang C-L, Peng J-P, Chen X-D. LncRNA-CIR promotes articular cartilage degeneration in osteoarthritis by regulating autophagy. Biochem Biophys Res Commun. 2018;505:692-8.

22. Filipowicz W. The widespread regulation of microRNA biogenesis, function and decay. Nat Rev Genet. 2010;11:597-610.

23. Paraskevopoulou MD, Hatzigeorgiou AG. Analyzing MiRNA-LncRNA interactions. Methods Mol Biol. 2016;1402:271-86.

24. Zhang S, Zhang JY, Lu L, et al. MiR-630 promotes epithelial ovarian cancer proliferation and invasion via targeting KLF6. Eur Rev Med Pharmacol Sci. 2017;21:4542-7.

25. Lu W, Zhang H, Niu Y, et al. Long non-coding RNA linc00673 regulated non-small cell lung cancer proliferation, migration, invasion and epithelial mesenchymal transition by sponging miR-150-5p. Mol Cancer. 2017;16(1):118.

26. Kim TH, Jeong JY, Park JY, et al. miR-150 enhances apoptotic and antitumor effects of paclitaxel in paclitaxel-resistant ovarian cancer cells by targeting Notch3. Oncotarget. 2017;8(42):72788-800.

27. Zhang Z, Wang J, Li J, et al. MicroRNA-150 promotes cell proliferation, migration, and invasion of cervical cancer through targeting PDCD4. Biomed Pharmacother. 2018;97:511-7.

28. Feng J, Yang Y, Zhang $P$, et al. miR-150 functions as a tumour suppressor in human colorectal cancer by targeting c-Myb. J Cell Mol Med. 2014;18(10):10.

29. Zhou QF, Tang XY, Tian XY, Tian J, Zhang Y, Ma J, Xu HX, Wang SJ. LncRNA MALAT1 negatively regulates MDSCs in patients with lung cancer. J Cancer. 2018;9:2436-42.

30. Xiong ZY, Wang LY, Wang QP, Yuan Y. LnCRNA MALAT1/miR-129 axis promotes glioma tumorigenesis by targeting SOX2. J Cell Mol Med. 2018;22:3929-40.

31. Li ZH, Xu L, Liu Y, Fu SK, Tu JH, HU YY, Xiong QY. LnCRNA MALAT1 promotes relapse of breast cancer patients with postoperative fever. Am J Transl Res. 2018;10:3186-97.

32. Wu Q, Meng WY, Jie Y, Zhao HJ. LncRNA MALAT1 induces colon cancer development by regulating miR-129-5p/HMGB1 axis. J Cell Physiol. 2018;233:6750-7.
33. Liang J, Xu L, Zhou F, et al. MALAT1/miR-127-5p regulates osteopontin (OPN)-mediated proliferation of human chondrocytes through PI3K/Akt pathway. J Cell Biochem. 2018;119(1):431-9.

34. Fernandes JC, Martelpelletier J, Pelletier JP. The role of cytokines in osteoarthritis pathophysiology. Biorheology. 2002;39:237-46.

35. Matyas JR, Adams ME, Huang D, Sandell L. Discoordinate gene expression of aggrecan and type II collagen in experimental osteoarthritis. Arthritis Rheum. 2014;38:420-5.

36. Eleonora Leucci FP, Johannes W, Kim H, Morten L, Bo P, Sakari K, Anders $\mathrm{HL}$. microRNA-9 targets the long non-coding RNA MALAT1 for degradation in the nucleus. Sci Rep. 2013;3:2535.

37. Chang SM, Hu WW. Long non-coding RNA MALAT1 promotes oral squamous cell carcinoma development via microRNA-125b/STAT3 axis. J Cell Physiol. 2018;233:3384-96.

38. Wu J, Weng Y, He F, Liang D, Cai L. LncRNA MALAT-1 competitively regulates miR-124 to promote EMT and development of non-small-cell lung cancer. Anticancer Drugs. 2018;29:628-36.

39. Yongqiang D, Guojun L, Bo Y, Chaoqun Y, Rui G, Xuhui Z. MALAT1 promotes the proliferation and metastasis of osteosarcoma cells by activating the PI3K/Akt pathway. Tumour Biol. 2015;36:1477-86.

40. Mende I, Malstrom S, Tsichlis P, Vogt P, Aoki M. Oncogenic transformation induced by membrane-targeted Akt2 and Akt3. Oncogene. 2001;20:4419-23.

41. Nakatani K, Thompson DA, Barthel A, Sakaue H, Liu W, Weigel RJ, Roth RA. Up-regulation of Akt3 in estrogen receptor-deficient breast cancers and androgen-independent prostate cancer lines. J Biol Chem. 1999;274:21528-32.

42. Editors TPO. Retraction: miR-122 regulates tumorigenesis in hepatocellular carcinoma by targeting AKT3. Plos ONE. 2017;12:e0184778.

43. Hu X, Wang J, He W, Zhao P, Ye C. MicroRNA-433 targets AKT3 and inhibits cell proliferation and viability in breast cancer. Oncol Lett. 2018;15:3998-4004

44. Liu Q, Qu X, Xie X, He P, Huang S. Repression of Akt3 gene transcription by the tumor suppressor RIZ1. Sci Rep. 2018;8:1528.

45. Wang $L$, Huang $D$, Jiang $Z$, et al. Akt3 is responsible for the survival and proliferation of embryonic stem cells. Biol Open. 2017;6(6):850-61.

46. Katsara $\mathrm{O}$, Attur M, Ruoff R, et al. Increased activity of the chondrocyte translational apparatus accompanies osteoarthritic changes in human and rodent knee cartilage. Arthritis Rheumatol. 2017;69(3):586.

47. Sakr M, Takino T, Sabit H, Nakada M, Li Z, Sato H. miR-150-5p and miR133a suppress glioma cell proliferation and migration through targeting membrane-type-1 matrix metalloproteinase. Gene. 2016;587:155-62.

48. Chen X, Xu X, Pan B, Zeng K, Xu M, Liu X, He B, Pan Y, Sun H, Wang S. miR$150-5 p$ suppresses tumor progression by targeting VEGFA in colorectal cancer. Aging. 2018;10:3421-37.

49. Okato A, Arai T, Kurozumi A, Kato M, Goto Y, Koshizuka K, Kojima S, Ichikawa T, Seki N. Dual-strands pre-microRNA-150 (miR-150-5p and miR-150-3p) act as tumor suppressor in prostate cancer. Cancer Res. 2017;51:245-56.

50. Ntoumou E, Tzetis M, Braoudaki M, et al. Serum microRNA array analysis identifies miR-140-3p, miR-33b-3p and miR-671-3p as potential osteoarthritis biomarkers involved in metabolic processes. Clin Epigenetics. 2017:9(1):127.

51. Xirui Y, Qi Z, Zhaomeng G, et al. Down-regulation of MiR-150 alleviates inflammatory injury induced by interleukin 1 via targeting Kruppellike factor 2 in human chondrogenic cells. Cell Physiol Biochem. 2018;47:2579-88.

\section{Publisher's Note}

Springer Nature remains neutral with regard to jurisdictional claims in published maps and institutional affiliations. 